\title{
Idosos institucionalizados e depressão: atividades expressivas e seu potencial terapêutico
}

\author{
Carolina Cangemi Gregorutti", Rita de Cássia Tibério Araújo**
}

\section{Resumo}

A rotina de vida do idoso nas instituições de longa permanência nem sempre inclui atividades direcionadas ao propósito lúdico e com aplicação sistematizada. O objetivo deste estudo foi introduzir uma programação de atividades expressivas e avaliar os seus efeitos no controle de sintomas depressivos manifestados por idosos institucionalizados. Participaram da pesquisa 7 idosos de uma instituição geriátrica que apresentavam sintomas sugestivos de depressão, tendo-se utilizado para essa avaliação a GSD-15, que foi aplicada ao início e ao término da programação terapêutica. A intervenção ocorreu durante quatro meses, realizando-se 2 sessões semanais de 75 minutos. Do total de 5 idosos com probabilidade de depressão no pré-teste, 3 atingiram a faixa de normalidade no pós-teste, recomendando-se a continuidade do estudo, para a ampliação da amostra e maior consistência dos resultados acerca dos efeitos das atividades expressivas sobre os sintomas depressivos manifestados por pessoas idosas.

Palavras-chave: Idosos. Instituição geriátrica. Depressão. Terapia ocupacional.

\section{Introdução}

$\mathrm{O}$ aumento da quantidade de idosos no Brasil tem gerado questionamentos quanto à qualidade de vida dessa população, havendo uma preocupação dos órgãos públicos no que se refere aos cuidados preventivos sob aspectos dos determinantes não somente biológicos, mas também relacionados aos ambientes de organização da vida coletiva. (BRASIL, 2006). A influência da condição emocional sobre a qualidade de vida na fase do envelhecimento é um tema que se destaca no âmbito das pesquisas, visto que as habilidades psicossociais da pessoa idosa são vulneráveis às mudanças que ocorrem na estrutura e função corporal e no ambiente. O agravo de doenças crônicas, o isolamento social, a viuvez, a aposentadoria com redução de ganhos, o afastamento dos filhos, a morte de familiares e amigos, a institucionalização, a solidão são alguns dos fatores agravantes para os sintomas depressivos. (CARVALHO; FERNANDEZ, 2002; CAMARANO et al., 2010).

\footnotetext{
* Graduada em Terapia Ocupacional pela Unesp - Marília. Mestranda pela Unesp - Marília. Endereço para correspondência: Rua Duque de Caxias, 147/74, Bairro: Montolar, CEP: 17502-580, Marília - SP. E-mail: carol.terapeut@gmail.com.

** Docente no curso de graduação em Terapia Ocupacional na Unesp - Marília. Docente no programa de Pós-Graduação em Educação, na linha de Educação Especial na Unesp - Marília.
}

$\longrightarrow$ doi:10.5335/rbceh.2012.025 
Gonçalves e Andrade (2010) relatam que a ocorrência de depressão está associada a fatores como idade, sexo, estado civil, condições sociais e atividades físicas. Diferentes estudos têm sugerido que o envelhecimento apresenta estreita relação com a presença de depressão, podendo a institucionalização agravar a condição depressiva. (CREUTZBERG; GONÇALVES; SOBOTTKA, 2008). Andrade et al. (2005), investigando a depressão em idosos institucionalizados, identificaram que a desesperança foi o sintoma mais prevalente. Conforme Vallillo (2004), todas as doenças são psicossomáticas, ou seja, começam como doenças emocionais e se transformam em doenças físicas e reais, como é o caso da depressão. Segundo o mesmo autor, a depressão no idoso, geralmente, ocorre depois que ele adquire patologias como câncer, Parkinson, demência, diabetes, juntamente com as perdas de pessoas queridas, com a diminuição de renda e com o fim da vida profissional e da fase reprodutiva.

A combinação de todos esses fatores, associados às mudanças na estrutura familiar, contribui para o aumento da demanda pela institucionalização de idosos em Instituições de Longa Permanência (ILPIs). (SOARES, 2010). A institucionalização dos idosos em países em desenvolvimento é uma situação que engloba condições socioeconômicas, educacionais e de saúde precárias. Não raramente, os idosos institucionalizados procedem de famílias com baixa renda, e as instituições, geralmente de cunho filantrópico, também não dispõem de recursos financeiros que possibilitem um cuidado mais adequado dessa população. (PERLINI; LEITE; FURINI, 2007). De acordo com Ballone (2002), a institucionalização, representando a subtração de um espaço existencial anteriormente vivido com plenitude e produção, pode provocar frustração. Complementando, Neri (2004) expõe que a institucionalização do idoso, associada às condições corporais e emocionais do envelhecimento, pode exacerbar os sintomas depressivos.

A institucionalização para a grande maioria dos idosos é fonte de dor e tristeza, o ambiente se torna silencioso, indiferente, vazio, passando a representar os momentos finais de sua vida podendo contribuir para o maior problema psicológico do idoso: a depressão. (PAMERLEE; KATZ; LAWTON, 1989).

Em contrapartida, Camarano et al. (2010) afirmam que viver em uma instituição pode representar uma alternativa de apoio e também de proteção e segurança. Optar por uma instituição não significa, necessariamente, uma redução da importância da família para o apoio e cuidado dos seus membros dependentes, mas uma nova organização e divisão de responsabilidades entre o Estado e o mercado privado. A institucionalização tampouco significa uma ruptura de laços familiares.

A questão que se apresenta neste estudo é a importância do cuidado com a programação e a organização de terapias que poderiam ser aplicadas nesse ambiente, visando à autonomia do idoso, uma vez que o prejuízo dessa condição constitui um risco de debilidade, à semelhança do que ocorre com pessoas que apresentam deficiência. (MCINTYRE; ATWAL, 2007). 
A autonomia significa a capacidade de reproduzir-se na complexidade da historicidade e da cotidianidade das mediações de poder, das energias e dos recursos próprios, na recusa da alienação, da tutela, do controle. Contudo, aquele que passa a residir em uma "instituição total", como são as instituições de longa permanência, passa a ser tutelado e controlado por diversos tipos de mecanismos que o impedem de tomar as decisões sobre seus desejos mais simples, como o horário de comer ou o que vestir. (GOFFMAN, 1989).

Uma alternativa para a modificação dessa situação seria o uso terapêutico de atividades que não só preencham o tempo ocioso, mas que também tenham um significado e contribuam para dar sentido ao seu cotidiano. Nessa perspectiva, a implantação de programas ocupacionais nas referidas instituições mostra-se essencial para a manutenção da qualidade de vida do idoso. (SOARES, 2010). Entre tais atividades, destacam-se, neste estudo, as atividades lúdicas.

Estudos têm demonstrado que o engajamento em ocupações correlacionam-se com a saúde de adultos idosos, com a função cognitiva e com o bem-estar subjetivo. (CLARKSON-SMITH; HARTLEY, 1990; ADELMANN, 1994). Contudo, são necessários mais estudos nessa linha para a ampliação do conhecimento e para documentar a importância da atividade na promoção da saúde.

As contribuições do engajamento da pessoa idosa em ocupações com significado lúdico são enfatizadas por Liberman (1998), que cita a importância do movimento sob a forma de atividades e oportunidades expressivas para viabili- zar o processo terapêutico ocupacional. Dentre as possibilidades de ações, este estudo aponta como alternativa o uso de atividades expressivas no cuidado a essa população, sendo objetivo desta pesquisa avaliar os efeitos dessa programação sobre o estado emocional de idosos institucionalizados.

\section{Método}

O projeto foi submetido ao Comitê de Ética em Pesquisa da Faculdade de Filosofia e Ciências da Unesp - campus de Marília e aprovado sob o Parecer n. 3.876/2008.

Trata-se de estudo exploratório com ênfase na função da ocupação, na medida em que esta favorece o bem-estar subjetivo. (CLARK; WOOD; LARSON, 2002). Participaram deste estudo 7 idosos institucionalizados, tendo-se como critérios de inclusão: capacidade cognitiva preservada, presença de sintomas sugestivos de depressão e condições clínicas adequadas à intervenção com base em registros de prontuário e informações médicas complementares. Ressalta-se que a conduta ética da pesquisadora, na condição de terapeuta ocupacional, levou a que fosse incluída na amostra uma participante com 55 anos de idade, a qual residia na instituição em que os dados foram coletados, manifestou interesse em participar da programação terapêutica e se enquadrava em todos os critérios de seleção, apresentando, principalmente, significativos sintomas sugestivos de depressão. 
A seleção dos participantes ocorreu, inicialmente, por indicação da assistente social da instituição, após esclarecimentos quanto ao perfil requerido para a constituição do grupo. Mediante a concordância, com a assinatura do Termo de Consentimento Livre e Esclarecido, e atendidas as demais providências dispostas na Resolução 196/96, os participantes foram submetidos ao Exame do Estado Mental (MEEM), que precedeu a aplicação da Escala de Depressão Geriátrica (GSD-15) - versão reduzida da GSD-30 de Yesavage e col. (1983), visando à definição da amostra consti- tuída por idosos sem prejuízo cognitivo importante e com sintomas sugestivos e/ ou indicativos de depressão.

Do total de 11 participantes indicados, 8 se enquadraram nos critérios de inclusão, e, com a desistência de 1 participante, a amostra ficou definida em 7 idosos. Na Tabela 1, encontram-se os dados dos participantes, a saber, idade, gênero e o escore obtido no MEEM. Os dados obtidos no pré-teste com relação aos escores da GSD-15 estão contemplados na Tabela 2, apresentada na seção de resultados.

Tabela 1 - Caracterização dos participantes.

\begin{tabular}{ccccc}
\hline Participantes & Idade & Gênero & $\begin{array}{c}\text { Escores } \\
\text { MEEM }\end{array}$ & Comprometimento cognitivo \\
\hline C.K & 55 anos & Feminino & 19 & Leve \\
J.M & 84 anos & Masculino & 19 & Leve \\
J.H & 70 anos & Masculino & 19 & Leve \\
K.S & 65 anos & Masculino & 29 & Sem comprometimento \\
P.L & 77 anos & Masculino & 23 & Leve \\
S.Z & 70 anos & Feminino & 24 & Leve \\
V.L & 74 anos & Feminino & 23 & Leve \\
\hline
\end{tabular}

A interpretação dos escores obtidos com a aplicação do MEEM foi elaborada com base nos seguintes critérios em termos de pontuação: 27 a 30 pontos $=$ sem alteração/comprometimento cognitivo, 19 a 26 pontos = alteração/ comprometimento cognitivo leve, 12 a 18 pontos = alteração moderada e 0 a 12 pontos = alteração/comprometimento cognitivo grave. No trabalho original de Folstein, Folstein e Mcnhugh (1975), o escore de 20 pontos (ou menos) era considerado o ponto de corte mais adequado para caracterizar pacientes com quadro de- mencial (ou comprometimento mental), independentemente de sua escolaridade. Considerando que a escolaridade é um fator importante para o desempenho no instrumento em questão e que no Brasil uma minoria dos idosos apresenta um alto nível escolar (IBGE, 2003), optou-se por utilizar, nesta pesquisa, a versão de Almeida e Almeida (1999), com os seguintes pontos de corte: $19 / 20$ para os que não possuem escolaridade e 23/24 para aqueles com histórico escolar prévio, conforme indicado acima. 
O estudo foi efetuado em uma cidade do interior do estado de São Paulo. A intervenção foi aplicada durante quatro meses, realizando-se 2 sessões semanais de 75 minutos cada. O programa de atividades englobou um conjunto de dinâmicas com ênfase na estimulação motora, cognitiva e psicossocial. Cada encontro recebeu um nome específico, de acordo com a atividade que seria desenvolvida. A seleção de atividades emergiu de discussões feitas no próprio grupo, não havendo, portanto, uma programação prévia, mas sim propósitos norteadores do processo terapêutico, os quais visaram a favorecer a oportunidade de relações interpessoais, de experiência em atividade grupal e de motivação para a prática de exercícios corporais com finalidade expressiva, como descrito no Quadro 1. Os registros de intervenção abrangeram dados de filmagens e anotações em diário de campo.

\begin{tabular}{|c|c|c|}
\hline Encontros & Objetivo & Resultado \\
\hline $\begin{array}{l}\text { Do } 1^{\circ} \text { ao } 7^{\circ} \\
\text { encontro: } \\
\text { Lembranças } \\
\text { (atividades com } \\
\text { música) }\end{array}$ & $\begin{array}{l}\text { Estabelecer vínculo } \\
\text { entre idoso e } \\
\text { terapeuta. } \\
\text { Exercitar a memória } \\
\text { recente e a antiga. }\end{array}$ & $\begin{array}{l}\text { Os participantes comentaram sobre suas profis- } \\
\text { sões de forma espontânea e relataram a necessi- } \\
\text { dade de se sentirem úteis novamente. }\end{array}$ \\
\hline $\begin{array}{l}\text { Do } 8^{\circ} \text { ao } 21^{\circ} \\
\text { encontro: } \\
\text { Divertimento (dança } \\
\text { e fotografia) }\end{array}$ & $\begin{array}{l}\text { Estimular as relações } \\
\text { interpessoais. }\end{array}$ & $\begin{array}{l}\text { Os participantes exteriorizaram os sentimentos } \\
\text { com relação à institucionalização, posicionando- } \\
\text {-se favoravelmente à decisão familiar, mas enfa- } \\
\text { tizando os aspectos negativos do isolamento e a } \\
\text { dificuldade para atribuir significados às suas vidas } \\
\text { num ambiente estranho, não familiar. Comenta- } \\
\text { ram sobre suas experiências amorosas e constru- } \\
\text { ções familiares. }\end{array}$ \\
\hline $\begin{array}{l}22^{\circ} \text { ao } 31^{\circ} \\
\text { Encontro: } \\
\text { Participação } \\
\text { (atividades de } \\
\text { exploração do } \\
\text { ambiente) }\end{array}$ & $\begin{array}{l}\text { Estimular a iniciativa } \\
\text { para o envolvimento } \\
\text { em atividades. }\end{array}$ & $\begin{array}{l}\text { Os participantes deram continuidade às ativida- } \\
\text { des com autonomia e desinibição, fotografando } \\
\text { os eventos grupais e o jardim da instituição, es- } \\
\text { crevendo poemas e letras de música. }\end{array}$ \\
\hline $\begin{array}{l}32^{\circ} \text { encontro: } \\
\text { A despedida } \\
\text { (encerramento das } \\
\text { atividades) }\end{array}$ & $\begin{array}{l}\text { Promover a } \\
\text { autoestima. }\end{array}$ & $\begin{array}{l}\text { Nesse encontro, o grupo teve a oportunidade de } \\
\text { reconhecer a importância do seu papel ativo no } \\
\text { engajamento institucional. }\end{array}$ \\
\hline
\end{tabular}

Quadro 1 - Programa de intervenção.

A análise dos dados coletados baseou-se no feedback dos participantes durante os encontros e na comparação dos resultados da GSD-15, obtidos ao início e ao término da intervenção. 


\section{Resultados}

Dos 7 participantes da pesquisa, 4 eram do gênero masculino e 3 do feminino.

Os resultados obtidos com a aplicação da GSD-15 indicam que, de todos os sujeitos pesquisados, $5(71,4 \%)$ apresentavam forte probabilidade para depressão e 2 (28,6\%), escores sugestivos de quadro depressivo (Tabela 2). A interpretação dos escores alcançados obedeceu aos seguintes critérios em termos de pontuação: 5 a 9 pontos = probabilidade de depressão e pontuação maior ou igual a 10 = depressão.

Após as 32 sessões, que totalizaram 40 horas de intervenção, observou-se que houve redução dos sintomas para todos os participantes, obtendo-se uma variação mínima de 1 ponto e máxima de 8 pontos (Tabela 2 ).

Tabela 2 - Variação de escores para depressão.

\begin{tabular}{ccc}
\hline $\begin{array}{c}\text { Escores } \\
\text { Pré-teste } \\
\text { (Pontos) }\end{array}$ & $\begin{array}{c}\text { Escores } \\
\text { Pós-teste } \\
\text { (Pontos) }\end{array}$ & $\begin{array}{c}\text { Escores } \\
\text { Variação } \\
\text { (Pontos) }\end{array}$ \\
\hline 6 & 4 & -2 \\
7 & 5 & -2 \\
6 & 5 & -1 \\
5 & 2 & -3 \\
6 & 2 & -4 \\
12 & 8 & -4 \\
14 & 6 & -8 \\
\hline
\end{tabular}

Do total de 5 idosos com forte probabilidade de depressão no pré-teste, três atingiram a faixa de normalidade de estado emocional no pós-teste. Por sua vez, os dois que apresentaram escores sugestivos de depressão no pré-teste baixaram para o limiar de indicativo de forte probabilidade de depressão.

Esses resultados, aliados ao registro de diário de campo, revelam que em um curto período de tempo (quatro meses) houve a formação efetiva de um grupo, fato que pôde ser constatado pela permanência espontânea de seus participantes, bem como pela fluidez de seus relatos e pela confiança estabelecida nas relações interpessoais, motivando-os a falar de seus sonhos, seus desejos, suas frustrações. Ao longo dos encontros, os registros de diário de campo e de filmagem revelaram aumento da frequência interativa entre os participantes e entre os participantes e o terapeuta. Também, foi constatado progresso na relação do participante com a atividade, comportamento revelado pela diminuição do tempo para o engajamento na atividade. Evidenciou-se, ainda, uma evolução positiva do grupo no âmbito da expressão corporal, comunicação verbal, interação grupal, motivação e iniciativa.

Tais resultados conferem mais clareza à compreensão da influência da ocupação sobre os estados subjetivos de bem-estar do adulto idoso, corroborando os dados da literatura e sinalizando os elementos individuais e grupais de possível causalidade da mudança do comportamento ocupacional no processo terapêutico. (CLARKSON-SMITH; HARTLEY, 1990; ADELMANN, 1994). 
Os resultados deste estudo apontam para a possibilidade de mudança das relações sociais nas instituições de longa permanência por meio de atividades que favoreçam o exercício expressivo, como recomenda Liberman (2008), sugerindo, também, que a atividade, ao preencher e promover a utilização do tempo, pode aliviar os efeitos da solidão. Levando em conta que a institucionalização e a solidão são fatores indutivos dos sintomas depressivos, conforme observação de Carvalho e Fernandez (2002), os resultados obtidos apontam, ainda, para a importância da ação terapêutica ocupacional no cuidado ao idoso institucionalizado.

\section{Conclusão}

Apesar de sua limitação, decorrente da amostra muito pequena e de possível viés amostral, o estudo pôde sugerir que a programação com atividades expressivas constitui uma possibilidade de intervenção terapêutica a ser rotineiramente trabalhada nas instituições geriátricas, na medida em que oferece probabilidade de alívio ou prevenção de sintomas depressivos. Nesta pesquisa, a aplicação do MEEM teve como propósito a seleção da amostra, conforme critério de inclusão, sugerindo-se, para novos estudos, a sua aplicação no pós-teste.
Institutionalized elderly and depression: expressive activities and their therapeutic potential

\section{Abstract}

The influence of emotional condition on quality of life during the aging process is a theme that stands out in the context of research, since the psychosocial skills of the elderly are vulnerable to changes in structure, function body and the environment. Given this, aim of this study was to evaluate the effects of a programming expressive activities in controlling depressive symptoms manifested by institutionalized elderly. Participants were 7 people in a geriatric institution with symptoms suggestive of depression and it was used to evaluate the GSD-15, which was applied at the beginning and end of treatment planning. The intervention was applied for four months, making up two weekly sessions of 75 minutes. Of the total of 5 seniors with probability of depression in the pretest, 3 reached the normal range for depression at post-test, recommending the continuation of the study to a larger sample and greater consistency of results about the effects of activities expressive about depressive symptoms manifested by older people.

Keywords: Elderly. Depression. Geriatric institution. Occupational therapy.

\section{Referências}

ADELMAN, P. K. Multiple roles and psychological well-being in a national sample o folder adults. Journal of Gerontology: Social Sciences, v. 49, n. 6, p. 277-288, 1994.

ALMEIDA, O. P.; ALMEIDA, S. A. Confiabilidade da versão brasileira da Escala de Depressão em Geriatria (GDS) versão reduzida. Arquivos de Neuro-Psiquiatria, v. 57, n. 2B, p. 421-426, 1999. 
ANDRADE, A. C. A. et al. Depressão em idosos de uma instituição de longa permanência (ILP): proposta de ação de enfermagem. Revista Gaúcha de Enfermagem, v. 26, n. 1, p. 57-66, 2005.

BALLONE, G. J. Alterações emocionais no envelhecimento. Revista PsiqWeb, 2002. Disponível em: <www.psiqweb.med.br >. Acesso em: 25 jan. 2008

BRASIL. Ministério da Saúde. Envelhecimento e saúde da pessoa idosa. Brasília/DF, 2006. (Cadernos de Atenção Básica, n. 19).

CAMARANO, A. A. et al. As instituições de longa permanência para idosos no Brasil. Revista Brasileira de Estudos Populacionais, Rio de Janeiro, v. 27, n. 1, p. 233-235, jan./ jun. 2010

CARVALHO, V. F. C.; FERNANDEZ, M. E. D. Depressão no idoso. In: PAPALÉO NETO, M. et al. (Org.) Gerontologia: a velhice e o envelhecimento em visão globalizada. São Paulo: Atheneu, 2002. p. 160-173.

CLARK, F.; WOOD, W.; LARSON, E. A. Ciência ocupacional: legado da terapia ocupacional para o século XXI. In: NEISTADT, M. E.; CREPEAU, E. B. (Org.). Terapia ocupacional. 9. ed. Rio de Janeiro: Guanabara Koogan, 2002. p. 10-17.

CLARKSON-SMITH, L.; HARTLHEY, A. A. The game of brigde as an exercise in working memory and reasoning. Journal of Geronto$\log y$, v. 45, n. 6, p. 233-238, 1990.

CREUTZBERG, M.; GONCALVES, L. H. T.; SOBOTTKA, E. A. Instituição de longa permanência para idosos: a imagem que permanece. Texto e Contexto - Enfermagem, Florianópolis, v. 17, n. 2, p. 273-279, 2008.

FOLSTEIN, M. F.; FOLSTEIN, S. E.; MCHUGH, P. R. Mini Mental Stabe: a practix al method for grading the cognitive status of pacients for the clinician. Journal Psychiatry Research, v. 12, n. 3, p. 189-198, 1975.

GOFFMAN, E. Manicômios, prisões e conventos. São Paulo: Perspectiva, 1989.
INSTITUTO BRASILEIRO DE GEOGRAFIA E ESTATÍSTICA (IBGE). Perfil dos idosos responsáveis pelos domicílios no Brasil. Rio de Janeiro, 2003. Disponível em: <http:// www.ibge.gov.br/home/estatistica/populacao/ perfilidoso>. Acesso em: 13 out. 2008.

LIBERMAN, F. Danças em terapia ocupacional. São Paulo: Summus, 1998.

MCINTYRE, A.; ATWAL, A. Terapia ocupacional na terceira idade. São Paulo: Santos, 2007.

NERI, A. L. Qualidade de vida na velhice. In: REBELATO, J. R.; MORELLI, J. G. S. (Org.). Fisioterapia geriátrica: a prática da assistência ao idoso. São Paulo: Manole, 2004. p. 1-28.

PAMERLEE, P. A.; KATZ, I. R.; LAWTON, M. P. Depression among institutionalized aged: assessment and prevalence estimation. Journal of Gerontology, v. 44, p. 22-29, 1989.

PERLINI, N. M. O. G.; LEITE, M. T.; FURINI, A. C. Em busca de uma instituição para a pessoa idosa morar: motivos apontados por familiares. Revista da Escola de Enfermagem da USP, São Paulo, v. 41, n. 2, p. 229-236, 2007.

SOARES, E. Projeto Memória e Envelhecimento: capacitando profissionais e aprimorando aspectos cognitivos em idosos institucionalizados. Revista Brasileira de Ciências do Envelhecimento Humano, Passo Fundo, v. 7, n. 1, p. 62-73, 2010.

VALLILLO, R. O mal do século atinge todas as idades. 2004. Disponível em: <http:// www.techway/revistaidoso/comportamento/ comportamentorenata.htm >. Acesso em: 20 out. 2010 .

YESAVAGE, J. A. et al. Development and validation of a geriatric depression screening scale: a preliminary report. Journal of Psychiatric Research, n. 17, v. 1, p. 37-49, 1983. 